\title{
The Cycle of the Shape Descriptor Suite: When do people become overweight?
}

\author{
David B. STEFAN ${ }^{1}$, David A. GILBERT ${ }^{2}$ \\ ${ }^{1}$ Novaptus Systems Inc., Chesapeake VA, USA; \\ ${ }^{2}$ The Hague Plastic and Cosmetic Surgery Center, Norfolk VA, USA \\ DOI: 10.15221/16.239 http://dx.doi.org/10.15221/16.239
}

\section{Background}

An enormous amount of anthropometric data has been collected over the last decade from using 3D body scanners to measure morbidly obese subjects prior to bariatric surgery. Even more anthropometric data has been collected by scanning these individuals post-operatively on a periodic basis. A set of shape descriptors and new adiposity indices were developed. These have been previously described and presented ${ }^{1,2}$. The shape descriptors mathematically classify the shape of the obese. The adiposity indicators use volume and surface units to define the degree of obesity by determining the amount of "space" occupied versus traditional weight and height and they are general in nature. When these formulas are combined and applied to scan images covering individuals ranging from visually thin to somewhat obese, an interesting phenomenon occurs. Thin individuals, regardless of gender, have similar shape descriptor values. As these formulas are applied to scans of individuals who appear slightly overweight to noticeably overweight, the shape descriptors begin to mathematically differentiate physical shapes and the ratio of torso volume and torso surface area rapidly changes. This paper investigates how the 3D adiposity indicators and the shape descriptors interact to detect the onset of excess weight that can lead to the obese condition.

\section{History of the 3D Adiposity Indicators}

In late 2002, the first 3D body scanner was installed in a cosmetic surgery clinic in Norfolk, VA. This particular scanner used white light mechanical projectors and 4 cameras, with one in each sensor head. The scanner footprint was a rather large $8 \times 16$ feet, and was placed in a storage room in the back of the clinic ${ }^{3}$.

A protocol was written and approved by an Institutional Review Board, and consent forms were developed, reviewed by the IRB and approved (Eastern Virginia Medical School, IRB \#05-09-FB0235). The purpose of the research was to utilize multidimensional information, such as volume and surface area, combined with linear and circumferential measurements provided by the scanner's measurement extraction software, and compare it to traditional measurement and photographic techniques employed by the cosmetic surgeon. The goal was to provide the cosmetic surgeon with new insights for viewing the contours of pre-operative patients. Measurements of periodic postoperative scans of a patient who underwent a body contouring procedure would be analyzed, evaluated and compared to the surgeon's expectations of the outcome.

Breast augmentation and breast reduction procedures were the first procedures to be analyzed. In a breast augmentation procedure, breast implants of known volume and dimensions are selected. The scanner was utilized as a 3D aid to breast contouring by attempting to measure and detect, among other things, any pre-operative breast asymmetries for which the surgeon could then take into account. Measurements of the patient's 3D whole-body model would also help to provide insight and recommendations into the proportionality of the breast and chest area with respect to the hips and waist, helping the surgeon and patient select the appropriately sized breast implants to complement overall body appearance ${ }^{4}$.

Other cosmetic procedures examined by the 3D scanner included lypoplasty, abdominoplasty and pannulectomies. Differences between preoperative measurements and post-operative measurements would be analyzed. Physical tissue that was removed would be weighed on a scale in grams, or fluid that was removed during a lypoplasty procedure would be collected and measured in cc. This was to be compared to changes in volume and surface area as measured by the scanner software.

In late 2005 , by chance, pre-operative surgical weight loss patients began to get scanned at the cosmetic surgery clinic. They received a printout of their common circumferential measurements. This was done voluntarily by the suggestion of a bariatric surgeon in a separate practice. After surgery, they would occasionally come back and get their post-operative scan, and would receive a print-out with the same measurements. In this way they could compare their pre-operative and post-operative measurements to use as an aid to monitor the changes their bodies were undergoing during the postsurgical weight loss period. 
We began to collect and compare multidimensional measurement information that the scanner provided in terms of surface area and volume. Among the scanner measurement files provided was a "torso slice file" that produced torso surface area in cm squared and volume in cc for each segment of what the scanner defined as a body model's torso.

The scanner software's definition of the torso extends from the "Back of the Neck" landmark determination to the "Crotch Point" landmark, that is, where it determines separation between the right and left legs and the torso. The difference in length between the "Back of Neck" landmark and the "Crotch Point" landmark is considered to be the "Torso Height." It can be measured in metric or English units. For our purposes, we use centimeters. The torso slice file output consists of $1 \mathrm{~cm}$ segments of torso surface area and torso volume, starting from the "Crotch Point" and terminating at the "back of neck" landmark. These slices are horizontal, and include the buttocks of the body model. Figure 1 depicts the torso as defined by the scanner software.

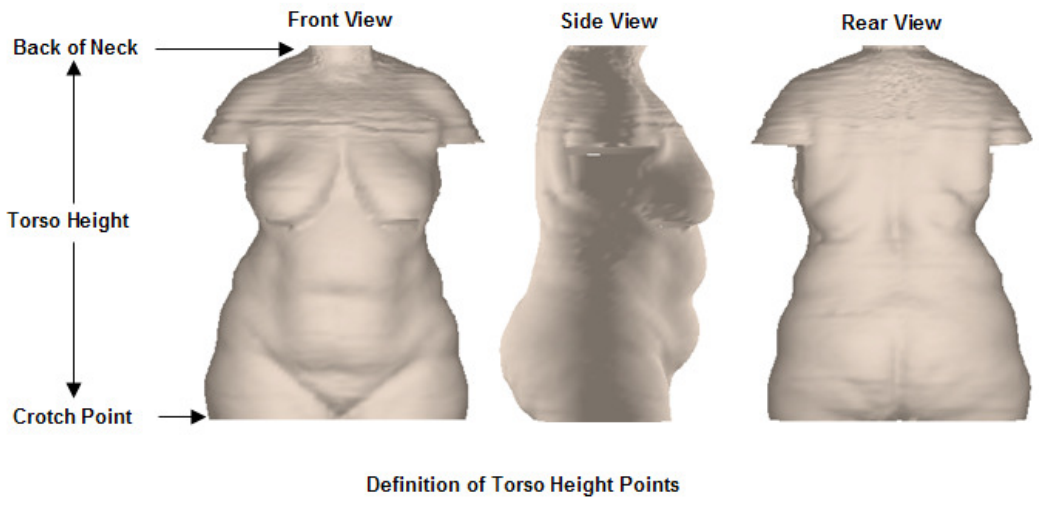

Figure 1. Definition of the Torso Height

Torso surface area and torso volume are each determined by summing up their respective segment values found along each $1 \mathrm{~cm}$ increment the torso height.

The top level analysis of these early scans as a whole gave little universal insight. An individual's series of pre- and post-operative scans allowed sequential multidimensional measurement tracking. Individuals that had the same procedure offered the possibility of longitudinal statistical analysis as a group.

It soon became apparent that the only thing that could be found in common amongst all the collected scans, and therefore all the individuals, was that everyone had a torso height. There were pre- and postoperative scan series for individuals who underwent breast augmentation, thereby increasing torso surface area and volume. And there were now pre- and postoperative scans series for individuals who underwent weight loss surgery, thereby reducing torso surface area and volume.

An investigation began into the relationship between torso height, torso surface area and torso volume.

It was found that there is a universal relationship between torso volume and torso surface area when the torso height is fixed, and it can be expressed by dividing the torso volume by the torso surface area. Specifically: The greater the "Torso Volume/Torso Surface Area" Ratio, the more obese the individual. Conversely, the lower the "Torso Volume/ Torso Surface Area" Ratio, the less obese the individual.

It can be said that the torso volume/torso surface area (TVSA) ratio determines the amount of "space" an individual occupies, without regard to a weight component.

One way to visualize the relationship between volume and surface area is to think about a single sheet of paper. It is very thin, but it has a large surface area (front and back) relative to the volume (thickness) that it possesses. As sheets of paper are stacked together, the volume (thickness) begins to increase at a more rapid rate relative to the stack of paper's surface area.

Thus the surface area of the torso acts like a "wrapper" around the volume of an individual's torso, following the contours of the torso's volume. The TVSA offers a general indication of the individual's physical shape, that is, only indicating how thin or obese an individual's torso may be. Figure 2 shows the different torso volume and torso surface area measurements for three individuals that have the same torso height of $64 \mathrm{~cm}$. 


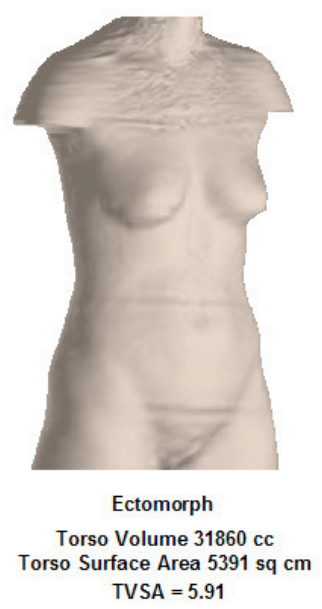

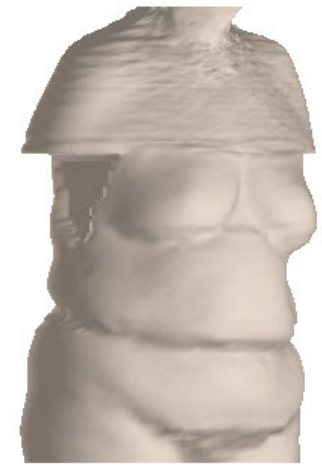

Endomorph

Torso Volume $55921 \mathrm{cc}$ Torso Surface Area $7135 \mathrm{sq} \mathrm{cm}$ TVSA $=7.84$

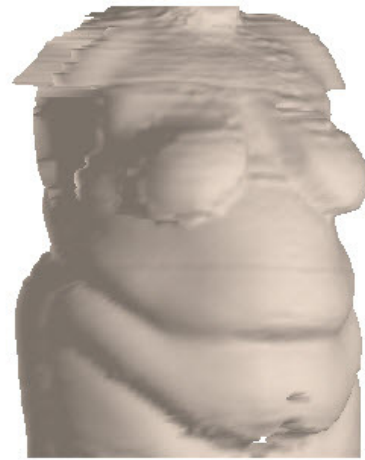

Morbidly Obese

Torso Volume $84858 \mathrm{cc}$ Torso Surface Area $8667 \mathrm{sq} \mathrm{cm}$ TVSA $=9.79$

Each Torso Height is $64 \mathrm{~cm}$

Figure 2. Shape of the Torso Determines Torso Volume/Torso Surface Area Value

The torso height component exists in every individual, and it is range bound in the adult population. Moreover, once an individual reaches the adult stage, their torso height can be treated as fixed for the great majority of their adult lives. This allows longitudinal comparisons of an individual's TVSA over time. There is some evidence that the torso height of an individual begins to decline slightly in their much older years, but this may be due to changes in posture or in some cases conditions like osteoporosis.

Individuals with the same torso heights can be directly compared to each other. Dividing the individual's TVSA by their torso height results in a dimensionless value that can be compared on a relative basis to the entire adult population. This value is called the Bariplex.

The analysis of scans for several thousand individuals has determined the range of values for torso height within the adult population, as well as the distribution. Figure 3 displays the range of torso heights recorded. To date, we have never recorded a torso height less than 57 or greater than $85 \mathrm{~cm}$ for the adults that have been scanned. The curve on the left is the distribution of torso height for females.

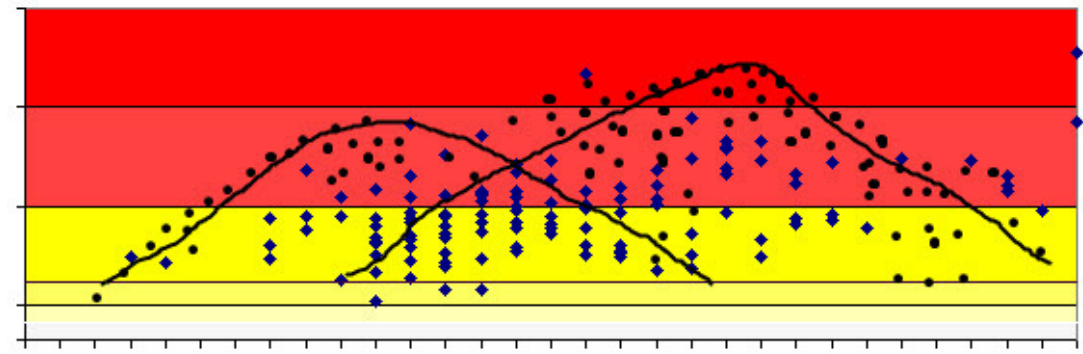

55565758596061626364656667686970717273747576777879808182838485

Torso Height, $\mathrm{cm}$

Figure 3. Observed Ranges of Adult Torso Heights

To determine an interpretive scale for the Bariplex, 200 body scans from the universe of scans collected between 2002 and 2005 were randomly selected. These included scans from the cosmetic surgery clinic, scans from a local fitness facility, and scans from the surgical weight loss volunteers. Both genders were included.

A statistical analysis was performed, and a distribution determined. The population was segmented into quintiles and the descriptions and cut-offs points were decided by committee. Both are subject to change if needed.

The original distribution was modified at the lower end as the number of scans of surgical weight loss individuals began to increase. Thus the current index reflects greater granularity between the obese, extremely obese and morbidly obese.

Figure 4 displays the Generalized Bariplex Scale as currently configured. 
The Generalized BariPlexsm Scale

\begin{tabular}{|c|c|c|}
\hline$\%$ & Bariplessm $^{\text {sm }}$ & Indication \\
\hline $\mathbf{9 1 - 1 0 0}$ & $\mathbf{1 2 . 5 9 6}$ & $\begin{array}{c}\text { Problematically } \\
\text { Thin }\end{array}$ \\
\hline $\mathbf{5 0 - 9 0}$ & $\mathbf{1 1 . 3 4 5}$ & $\begin{array}{c}\text { Proportionally } \\
\text { Ideal to Thin }\end{array}$ \\
\hline $\begin{array}{c}\text { Less than } \\
\mathbf{5 0}\end{array}$ & $\mathbf{9 . 8 1 4}$ & $\begin{array}{c}\text { Slightily } \\
\text { Overweight }\end{array}$ \\
\hline $\begin{array}{c}\text { Less than } \\
\mathbf{4 0}\end{array}$ & $\mathbf{9 . 4 7 0}$ & 0verweight \\
\hline $\begin{array}{c}\text { Less than } \\
\mathbf{3 0}\end{array}$ & $\mathbf{8 . 9 4 4}$ & 0hese \\
\hline $\begin{array}{c}\text { Less than } \\
\mathbf{2 0}\end{array}$ & $\mathbf{8 . 5 1 8}$ & Extremely Ohese \\
\hline $\begin{array}{c}\text { Less than } \\
\mathbf{1 0}\end{array}$ & $\mathbf{7 . 7 8 3}$ & Morbinlly Ohese \\
\hline
\end{tabular}

Figure 4. The Generalized Bariplex Scale.

\section{History of the Shape Descriptors}

The Bariplex appears to be a valid adiposity indicator, differentiating the degree of obesity by determining the "space" individuals occupy rather than relying solely on height and weight, as is the case with BMI.

However, the Bariplex, or its counterpart the TVSA, like BMI, does not give any description of the shape of the individual. One can generally look at an individual and visually estimate the distribution of fat. There were no mathematical formulas that utilized the plethora of multidimensional information provided by the 3D scanner to calculate the body shape of the obese.

In weight loss surgery, the morbidly obese body shape is generally classified between two extremes. On one end is the "gynecoid" shape, known colloquially as the "pear" shape. On the other end is the "android" shape, commonly considered to be an "apple" shape. Individuals that possess these two shapes are readily identifiable. However, there exists a wide range of shapes between these two extremes. A majority of individuals that present for weight loss surgery are neither strictly "gynecoid" or strictly "android." These shapes defy common descriptive terms, such as "pearish," or "applish." The general term "mixed shape" is often applied to these individuals, but this description, by its very nature, is no definite shape description at all.

Individuals that possess a pear shape tend to have their excess fat centered around their hips and buttocks area. They often have large hip sizes, large buttocks and large thigh measurements associated with a relatively smaller waist measurement. Individuals that possess an apple shape tend to have their excess fat centered around their waist, stomach and abdomen. They often have proportionally smaller hips, buttocks and thigh measurements than their pear-shaped counterparts. In general, those with an android shape are considered to be at more risk to develop co-morbid conditions associated with morbid obesity. Figure 5 depicts 3D scan images of those who have shapes that are considered android and gynecoid.

\section{Android vs. Gynecoid}
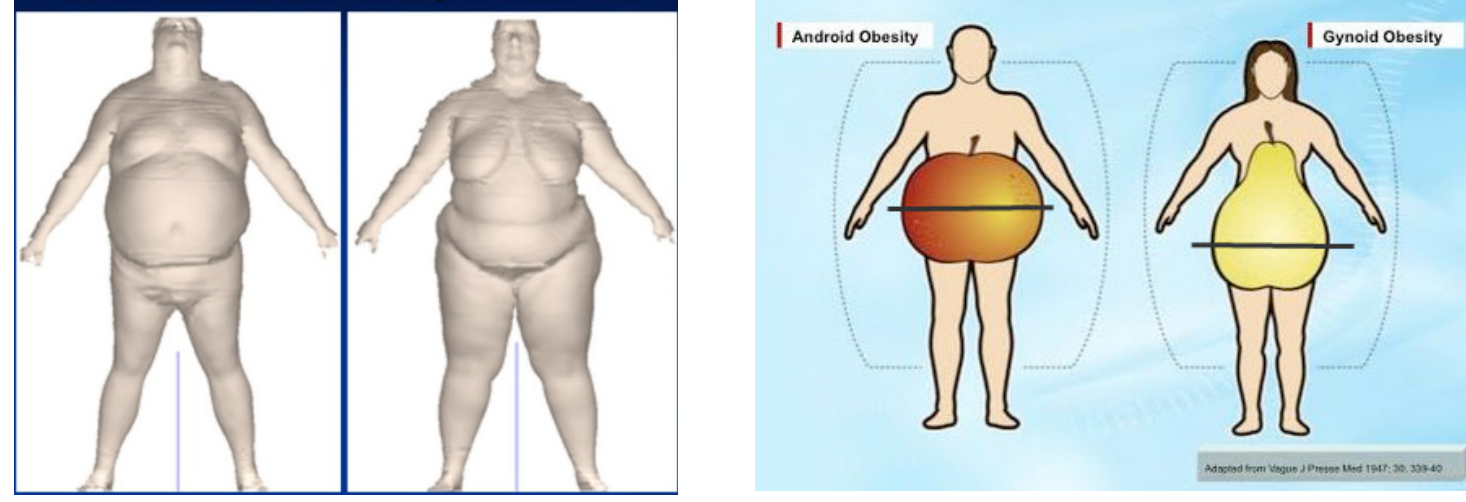

Figure 5. Example Excess Weight Distribution for Android and Gynecoid Body Shapes 
Enter the torso slice file, again. Recall that the torso slice file segmented the body model's torso into horizontal slices. In our case, these slices were in centimeters. Associated with each slice is the height of the slice, from the floor, and the circumference of the slice. Since each torso segment is 1 $\mathrm{cm}$ in height, one can easily determine the segment's surface area. By "closing" the segment, its volume in cc can be determined.

The torso slice file can be searched for the maximum torso circumference. Presumably, this is the segment on the torso that contains the area of maximum fat distribution. Once found, the maximum torso height point of that torso segment can be determined.

By determining the individual's overall torso height, and the height of the maximum torso circumference, a mathematical determination of the subject's shape can be calculated. This is done by dividing the torso volume/torso surface area product from the back of the neck down to where the height of the maximum torso circumference into the overall torso volume/torso surface area of the torso.

The theory behind this is straightforward. The overall torso volume/torso surface area ratio indicates that amount of "space" an individual occupies, given their torso height. If the individual is thin, the torso surface area will be proportionately higher than the corresponding torso volume and will result in a lower TVSA value. If the individual is obese, extremely obese or morbidly obese, their torso volume will be proportionately greater than their torso surface area, and their TVSA value will be higher.

The location of the maximum torso slice height is where the distribution of fat most likely is the greatest. Thus dividing the top torso volume/torso surface area above and including the maximum torso volume/torso slice circumference height by the overall torso volume/torso surface area always yields a number between 0 and 1 . If the number is nearer to 1 , the obese individual has the majority of the excess weight centered around their hips and buttocks area. If the number is nearer to 0.5 , it means that the excess weight is centered around their waist/stomach/abdomen area. Figure 6 outlines the concept of the determining the primary shape of the torso using this method.

\section{Primary Shape Determination}

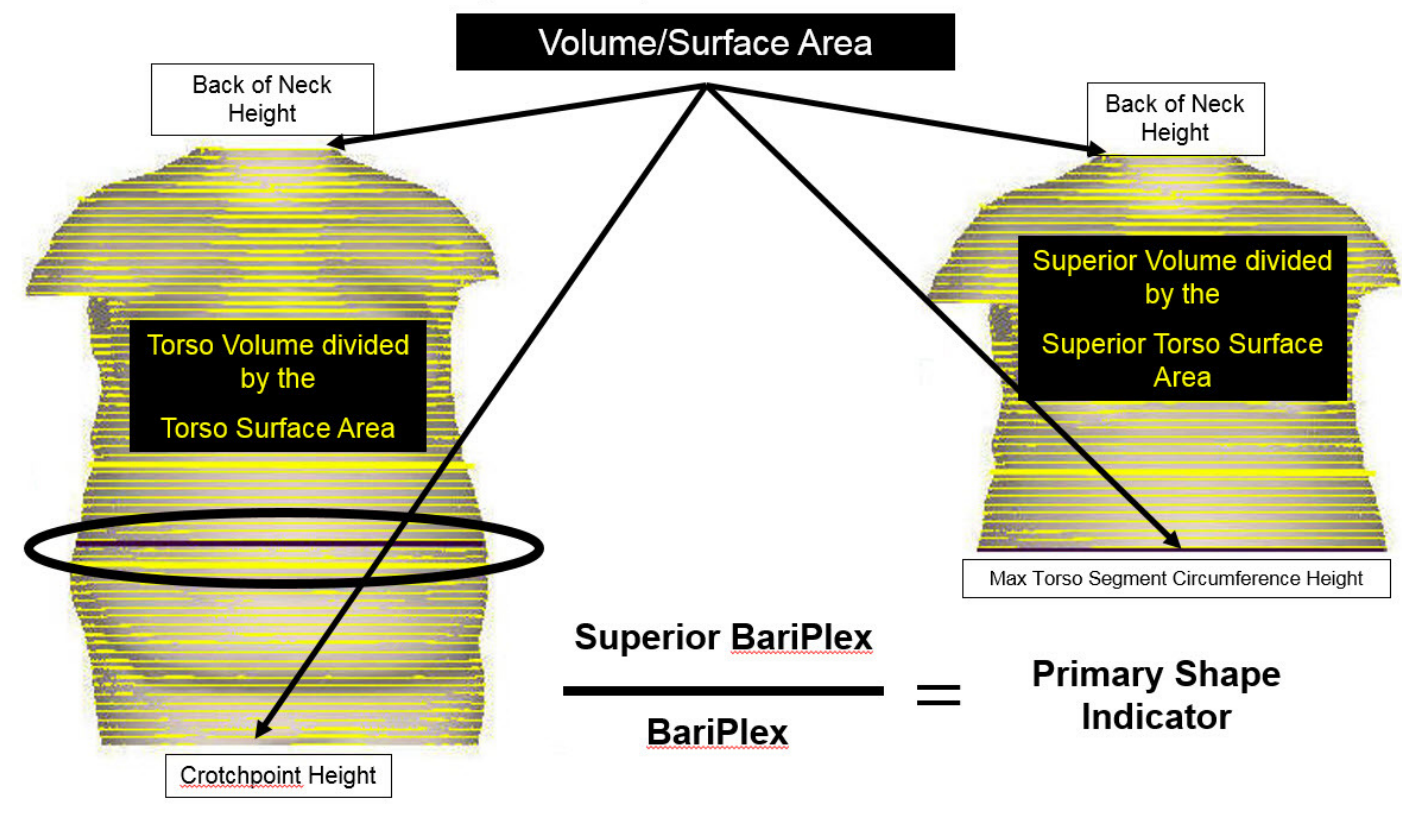

Figure 6. Primary Shape Determination

This shape descriptor, known as the Primary Shape, has been applied to thousands of morbidly obese individuals before and after their weight loss surgery. In practice, it has been found that the great majority of Primary Shape values are concentrated between 0.7 and 0.8. A Primary Shape Descriptor Scale was developed with the aid of practicing bariatric surgeons. It was determined that anyone possessing a Primary Shape of 0.8 or higher was considered gynecoid in shape. Anyone possessing a Primary Shape of 0.7 or lower was considered an android shape. In practice, we have recorded android shapes as low as 0.55 , and gynecoid shapes as high as 0.91 , among the obese. 
Those with a Primary Shape value between 0.71 and 0.79 have a shape that considered as mixed, with the visual definition of a mixed shape being 0.75 . Thus someone with a Primary Shape of 0.78 was considered to have a mixed shape that tended toward being gynecoid. Someone with a Primary Shape value of 0.73 was considered to have a mixed shape that tended toward being android. Figure 7 displays how to interpret the Primary Shape value.

\section{PRIMARY SHAPE \\ DETERMINATION}

\begin{tabular}{|c|c|}
\hline$\geq 0.80$ & Gynecoid \\
\hline $0.71-0.79$ & Mixed \\
\hline$\leq 0.70$ & Android \\
\hline
\end{tabular}

Figure 7. Primary Shape Interpretation

To further refine the definition of shape, a secondary indicator was calculated. This secondary indicator was called the Shape Tendency. It has been described in previous papers and presentations and is only of concern to refine the shape definition of the morbidly obese and extremely obese and will not be discussed here ${ }^{5}$.

The combination of the TVSA and the Shape Descriptors have proven to be an effective complement, but not an entire replacement for height and weight when applied to the obese. Together, they define the degree of obesity and give an indication of the weight distribution. Of course, the height and weight of the individual are and will always be important components of the overall evaluation. This is particularly true when tracking weight loss associated with bariatric surgery by periodic post-operative scanning, comparing measurements changes, including volume and surface area information, alongside the more familiar common circumferential measurements.

Weight loss surgery, particularly a gastric bypass or gastric sleeve procedure generally results in massive weight loss over a period of 1 year. It is not uncommon for individuals who undergo such procedures to lose up to $75 \%$ of their excess body weight. For many, this means the possibility of losing up to $50 \%$ of their preoperative weight, sometimes more.

A very interesting phenomenon was noticed about the Primary Shape Descriptor value as applied to the morbidly obese who underwent surgical weight loss. The Primary Shape value was calculated before their surgical procedure, and sequentially after their procedure as they returned for follow-up appointments on a routine basis. These individuals progressively lost a great deal of weight, with the largest amount weight loss generally occurring within the first 3 months. Significant weight loss continued after the first 3 months, albeit at a slower rate as recorded at the 6 month and yearly scan interval.

It was noticed that the pre-operative Primary Shape value of the weight loss surgery candidate did not change significantly during the post-surgical weight loss process. This was astonishing. These individuals literally lost "half a person," yet their shape, as calculated by the Shape Descriptor formula remained tightly range-bound throughout the weight loss process.

Even though individuals who underwent weight loss surgery often achieved the desired goal of "75\% excess weight loss," very few of these surgical weight loss procedures resulted in these individuals eventually possessing a Bariplex value that was, according to the scale, "Proportionately ideal to thin." Instead, despite the massive weight loss most ended up in the "overweight" or even the "obese" Bariplex categories.

Of those weight loss surgery individuals fortunate enough to lose enough weight to enter the "Proportionately ideal to thin" segment of the Bariplex scale, another Shape Descriptor phenomenon occurred. Toward the end of their weight loss period, the Shape Descriptor value ceased to function, regardless of their consistently similar previous values. These individuals did not appear to have any excess fat anymore and were visually thin. 
This indicated that at some point in the weight loss process, as the shape of these individuals went from obese to "thin," the Shape Descriptors, designed specifically for determining the shape of the overweight, broke down and became ineffective. This was true for both male and female individuals.

It was at this point that an investigation began to take place regarding the behavior of the Primary Shape descriptor. Was it possible to use the Shape Descriptor formulas in reverse? That is, is there a particular torso volume/torso surface area value that triggers the Primary Shape Descriptor into activity? Could this technique then be used to monitor and alert individuals who start to become overweight, rather than being used exclusively for those that have already become obese and are undergoing weight loss?

\section{Methods}

In an attempt to discover the lower bounds of the Primary Shape Descriptor function, the formulas were applied to body models of scans taken of visually "thin, normal, slightly obese and obese" individuals. The sample set used for this analysis contained 44 individuals who volunteered to be scanned from two local fitness facilities. 17 were female, 27 were male.

The population ranged from very short in stature, to very tall as measured by the torso height. And from extremely thin to extremely obese. The population of men skewed toward being tall and visually thin and well-proportioned as many who were scanned were bodybuilders.

The summary statistics for the entire population in Table 1.

\begin{tabular}{|c|c|c|c|c|c|}
\hline & $\begin{array}{c}\text { Torso Height } \\
\mathrm{cm}\end{array}$ & TVSA & Bariplex & Shape & $\begin{array}{c}\text { Mid-Max Dist. } \\
\mathrm{cm}\end{array}$ \\
\hline Median & 72 & 6.91 & 10.13 & 0.91 & 26 \\
\hline Low & 57 & 5.5 & 6.51 & 0.57 & 5 \\
\hline High & 80 & 10.28 & 12.21 & 1.00 & 32 \\
\hline Average & 71 & 7.14 & 10.10 & 0.87 & 24 \\
\hline STD DEV & 6 & 1.00 & 1.25 & 0.10 & 7 \\
\hline
\end{tabular}

Table 1. Summary Statistics for Male and Female Sample Population

The population summary statistics indicate a general mix of height and shape. The shortest individual had a torso height of $57 \mathrm{~cm}$. The tallest individual had a torso height of $80 \mathrm{~cm}$.

The most obese individual had a Bariplex of 6.51 , which is considered to be morbidly obese. This was a female. Her Primary Shape descriptor was 0.8, indicating a gynecoid shape whereby the preponderance of excess weight is distributed around the hips and buttocks area. The thinnest individual had a Bariplex of 12.21. This was a tall, thin male. The Primary Shape indicator for him was 1.0 , indicating that the formula ceased to function.

The average Bariplex, which is the TVSA normalized by dividing by the TVSA by the Torso Height was 10.10 , very close to the median. A glance at the Generalized BariPlex scale in Figure 4 indicates, as a whole, the general population was considered to be "Proportionally ideal to thin". However, there were some subjects that had a Bariplex value that fell into the extremely obese, obese and slight obese categories.

\section{Analysis of Male Population}

The sample population was divided by gender. The summary statistics for the male population are found in Table 2.

\begin{tabular}{|c|c|c|c|c|c|}
\hline & $\begin{array}{c}\text { Torso Height } \\
\text { cm }\end{array}$ & TVSA & Bariplex & Shape & $\begin{array}{c}\text { Mid-Max Dist. } \\
\text { cm }\end{array}$ \\
\hline Median & 75 & 6.96 & 10.32 & 0.92 & 27 \\
\hline Low & 68 & 6.32 & 8.59 & 0.57 & 5 \\
\hline High & 80 & 8.38 & 12.21 & 1.00 & 32 \\
\hline Average & 75 & 7 & 10 & 1 & 24 \\
\hline STD DEV & 3 & 1 & 1 & 0 & 8 \\
\hline
\end{tabular}

Table 2. Summary Statistics for the Male Subpopulation 
Example ranges of male shapes and sizes that were analyzed are arrayed in the Figure 8.

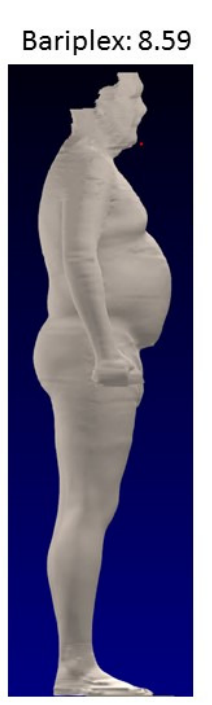

Extremely Obese
Example of the Range of Male Shapes and Sizes Analyzed
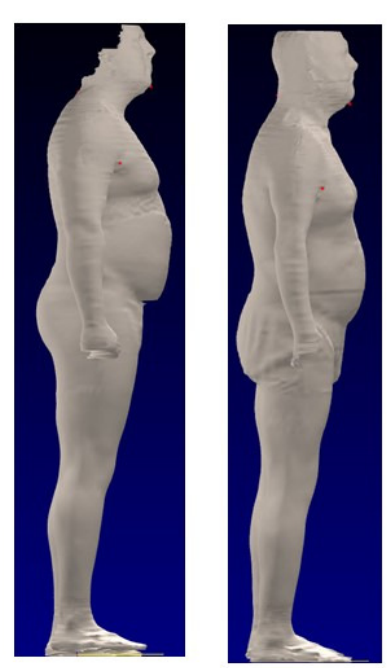

Obese

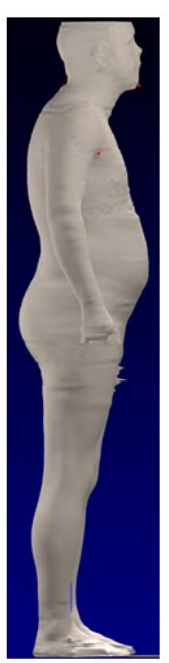

Overweight
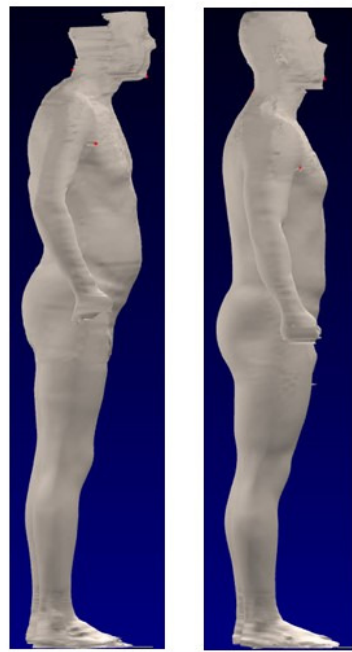

Proportional Thin
Bariplex 12.21
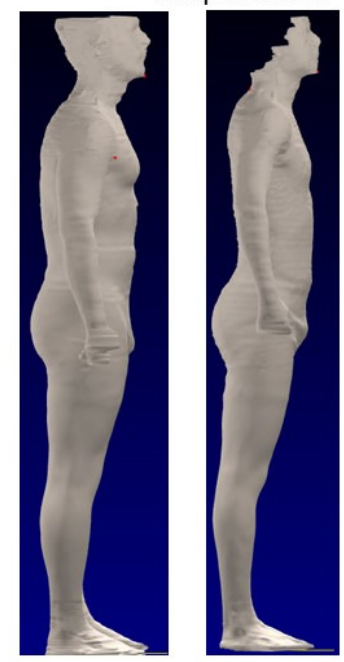

Very Thin

Figure 8. Representative Shapes for the Male Sample Population

For illustrative purposes they have been scaled to appear as if they were all the same height. In actuality, there are some variances in individual torso heights, as per the normal population. The obese examples shown toward the left are instances of centralized obesity, as determined by their primary shape value. Shapes of those on the left are considered to be "android or tending android." There are also examples in the male sample population that are considered obese and "gynecoid" in shape. These are not shown in Figure 8, but are included in the analysis.

A man that is thin by nature, or "well-proportioned" as a result of an exercise regimen does not necessarily have any excess fat. There are several examples of such individuals within this population. These individuals have a similar appearance as the males depicted in the far right of Figure 8. In these cases, application of the Shape Descriptor formula results in the individual possessing a shape that would be considered "extreme" gynecoid. However, that term isn't applicable to their body shape as the term "gynecoid" implies a degree of obesity.

\section{Analysis of "Proportional and Thin" males}

The male population was further segmented by Bariplex. According to the interpretive scale, someone possessing a Bariplex value of 10.0 or higher should be classified as "Proportionally thin, thin or extremely thin." Males with a Bariplex of 10.0 or higher are summarized in Table 3.

\begin{tabular}{|c|c|c|c|c|c|}
\hline Thin & $\begin{array}{c}\text { Torso Height } \\
\text { cm }\end{array}$ & TVSA & Bariplex & Shape & $\begin{array}{c}\text { Mid-Max Dist. } \\
\mathrm{cm}\end{array}$ \\
\hline Median & 76 & 6.83 & 10.755 & 0.93 & 29 \\
\hline Low & 69 & 6.32 & 10.05 & 0.63 & 9 \\
\hline High & 80 & 7.91 & 12.21 & 1 & 32 \\
\hline Average & 75 & 6.89 & 10.88 & 0.92 & 27 \\
\hline STD DEV & 3 & 0.45 & 0.63 & 0.09 & 6 \\
\hline
\end{tabular}

Table 3. Summary Statistics for Male Subpopulation having Bariplex Value of 10.0 or Greater

Inspection of the above table indicates that the average shape of the males in this group was 0.93 . This would be consistent with a thin male having little excess fat distribution. There is one instance of a male that, although the Bariplex value indicated a thin torso, possessed a shape indicator pointing towards an extreme android shape of 0.63 .

This man had a torso height of $77 \mathrm{~cm}$. He is depicted in Figure 9 in side view with another male that has the same torso height, but a shape indicator of .93. 


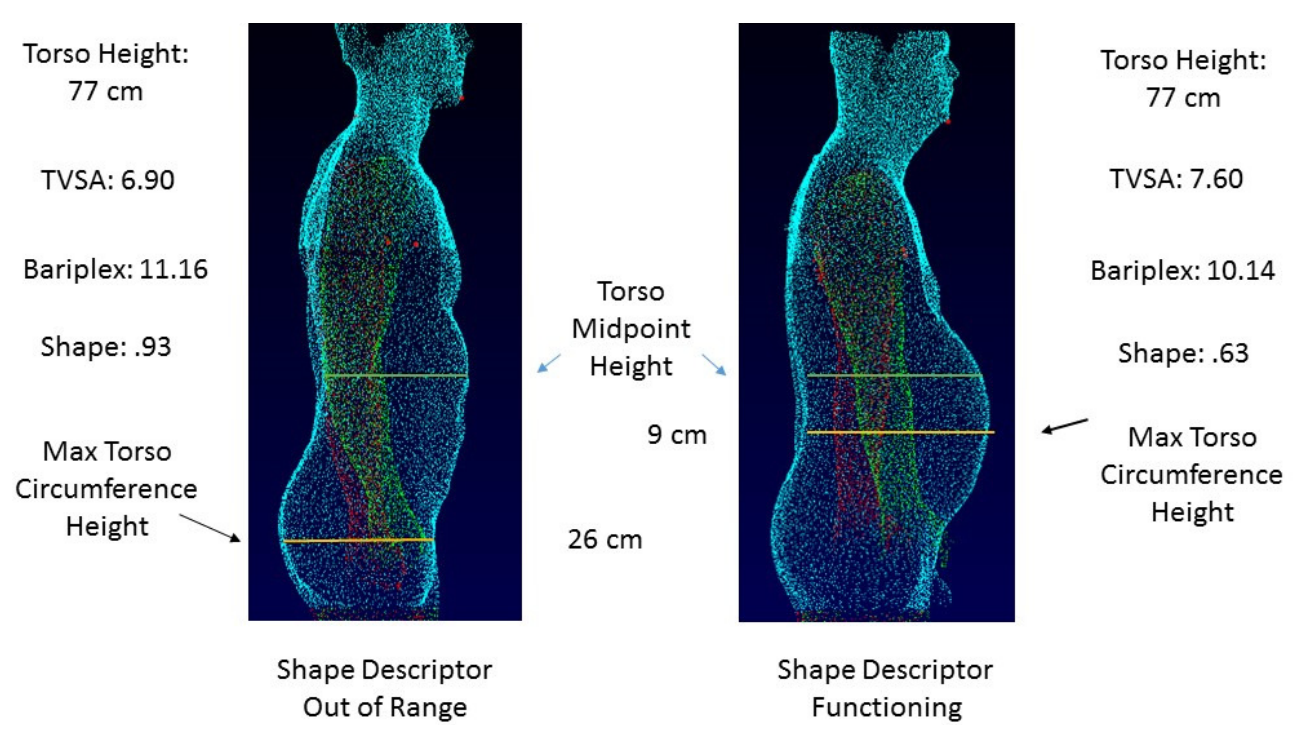

Figure 9. Two Males with Same Torso Height and Different Torso Shapes

If one inspects the torso slice file output of a thin male body model it will be found that the maximum torso circumference occurs near or below the torso slice height that represents the area around or below the hips measurement location. The mathematics are such that the surface area of this type of body model is proportionately greater than its volume, hence a low TVSA number (6.90) is the result. If one was to inspect his common circumferential measurements, it would be found that the hips circumference is larger than his waist, stomach and abdominal circumferential measurements.

Applying the shape descriptor formula to such a body model often results in a primary shape value of 0.90 to 1.0. This means that the torso volume/torso surface area from the back of the neck down to the maximum torso height circumference is the same or nearly the same as the overall TVSA associated with the torso height. When one is divided into the other, the result tends towards 1.0. The shape descriptor formula does not detect any excess weight distribution.

The torso volume of the man on the right is proportionally higher than his torso surface area, resulting in a higher TVSA, despite the two men having the same torso height. The shape descriptor formula found that his maximum torso circumference height was closer to the midpoint of his torso, indicating excess fat distribution in the abdominal area. This is further corroborated by the decreased distance between the man's torso midpoint height and the height of the maximum torso circumference (shown in the table as 'Mid-Max').

The man on the right is not considered to be thin, even though his Bariplex value places him in such a category. This is an important example in that the Bariplex, like BMI cannot be relied upon alone to judge the weight distribution of an individual since there is no shape information provided. The addition of the Shape Descriptor detected a centrally obese condition that may warrant further investigation. If this is so, it might also have been possible to detect the onset of such a condition using $3 \mathrm{D}$ body scanning techniques and these equations.

In the sample set of males, the combination of a Bariplex above 10, a shape descriptor value of .90 or above categorically indicates a thin or proportionally well-built man. The mid-max distance is a secondary consideration in that it takes into account the torso height of the individual. Men that meet the Bariplex criteria and shape criteria mentioned above will also be thin, but due to possessing a shorter torso, the mid-max distance will may not be as great as thin men who have taller torsos found in the sample population that was analyzed.

However, for a given torso height, the lower the mid-max distance number the more centrally obese the individual.

\section{Analysis of Male Slight to Overweight "Borderline" Examples}

Individuals that have a Bariplex number of below 10.0 can begin to exhibit areas of excess fat distribution. The relationship between their torso volume and torso surface area is such that their torso volume is starting to become proportionally larger than their torso surface area. Though these individuals appear to be not as thin as those with higher Bariplex values, they are not necessarily 
visually obese. However, they may be trending in a particular direction. This is the grey area between "in shape," and slightly overweight and overweight. Periodic scanning of these individuals, and the determination of their Bariplex and Shape Descriptor value at the time of each particular scan could yield actionable trend information. The summary statistics for this group are found in Table 4.

\begin{tabular}{|c|c|c|c|c|c|}
\hline $\begin{array}{c}\text { Borderline } \\
\text { Overweight }\end{array}$ & $\begin{array}{c}\text { Torso Height } \\
\mathrm{cm}\end{array}$ & TVSA & Bariplex & Shape & $\begin{array}{c}\text { Mid-Max Dist. } \\
\mathrm{cm}\end{array}$ \\
\hline Median & 77 & 7.79 & 9.8 & 0.92 & 26 \\
\hline Low & 68 & 6.94 & 9.73 & 0.77 & 21 \\
\hline High & 77 & 7.91 & 9.88 & 1 & 30 \\
\hline Average & 74 & 7.55 & 9.80 & 0.90 & 26 \\
\hline STD Dev & 4 & 0.43 & 0.06 & 0.10 & 4 \\
\hline
\end{tabular}

Table 4. Summary Statistics for Male Subpopulation having Bariplex Value of 9.5 to 9.99

There are two examples of note.

As indicated in the above chart, an individual in the "borderline" category had a shape value of "1." This indicates that there was no excess fat and that he was "proportionally well built." Another individual had a shape value of 0.77 , indicating some shape distortion, particularly around and above his hips area.

Figure 10 displays these two individuals. As is visually evident, the male on the left who has a Primary Shape value of 1 appears to be well proportioned and physically fit and does not indicate any excess fat distribution. The male on the right appears to be slightly overweight, with the excess fat distributed between the hips area and the waist area as indicated by his shape value. They have different torso heights so their TVSA cannot be compared directly with one another, rather, their Bariplex value must be used for comparison.

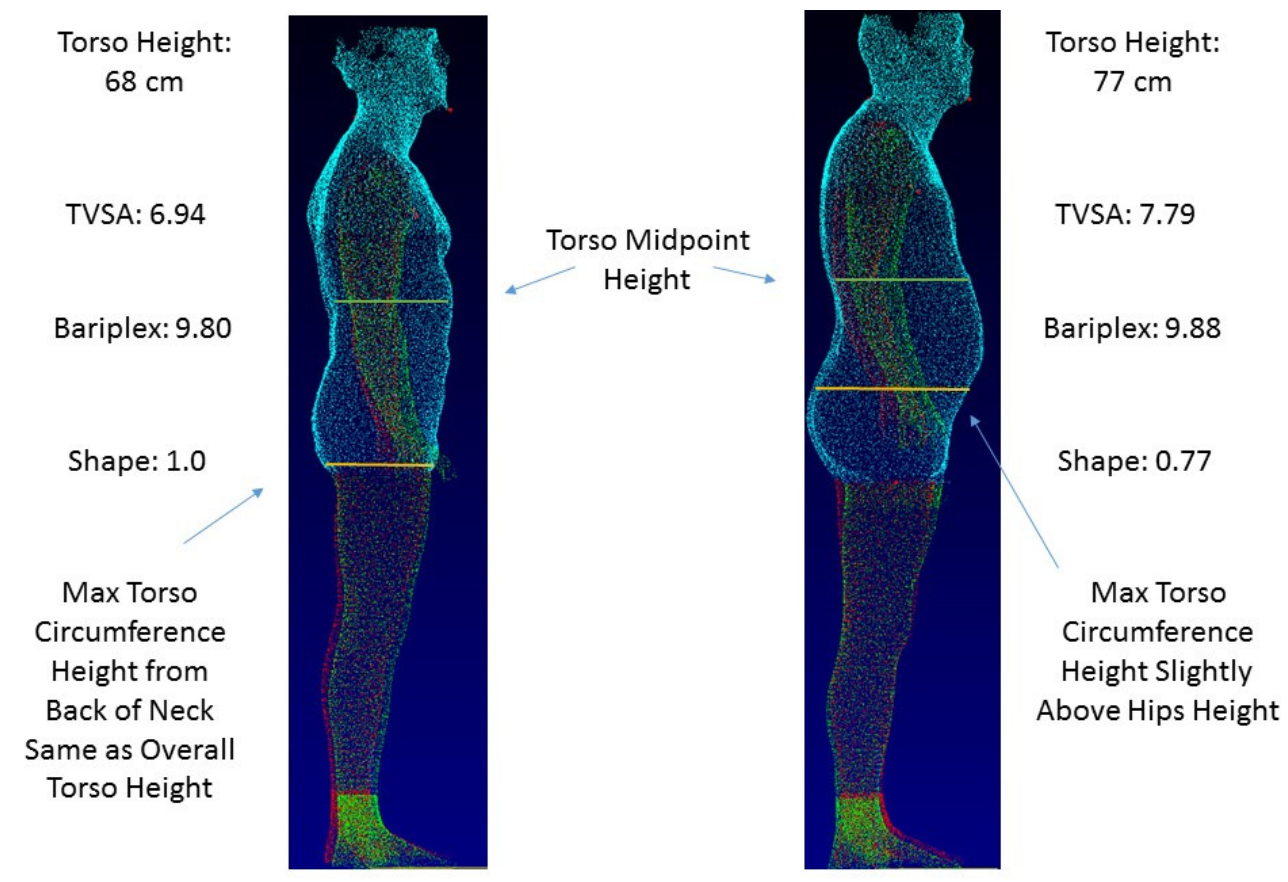

Figure 10. Two Males with Similar Bariplex Values but Different Body Shapes

Here we can clearly view the difference in shape between the two males that have similar Bariplex values. The one on the left is physically fit. The combination of the Bariplex and the Shape Descriptor can act as an aid to discriminate between the physically fit and those who are on the borderline between "normal" weight and overweight. 


\section{Analysis of Overweight Male Examples}

A Bariplex below 9.5 indicates an overweight condition. The lower the Bariplex value, the more obese the individual. The shape descriptor value in this case gives an indication of where the excess weight is distributed along the torso. Table 5 provides summary statistics for the males in the sample set that had a Bariplex of less than 9.50.

\begin{tabular}{|c|c|c|c|c|c|}
\hline Obese & $\begin{array}{c}\text { Torso Height } \\
\mathrm{cm}\end{array}$ & TVSA & Bariplex & Shape & $\begin{array}{c}\text { Mid-Max Dist. } \\
\mathrm{cm}\end{array}$ \\
\hline Median & 75 & 8.205 & 9.25 & 0.71 & 13 \\
\hline Low & 70 & 7.79 & 8.59 & 0.57 & 5 \\
\hline High & 79 & 8.38 & 9.49 & 0.91 & 29 \\
\hline Average & 74 & 8.16 & 9.11 & 0.74 & 15 \\
\hline STD DEV & 3 & 0.20 & 0.39 & 0.13 & 8 \\
\hline
\end{tabular}

Table 5. Summary Statistics for Male Subpopulation having Bariplex Value below 9.5

All who possessed a Bariplex value in this category were visually obese. Their average shape indicated a "mix-nature, tending android" value of 0.74 . but there was an example of an extreme gynecoid shape (0.91) and an extreme android shape (0.57). Figure 11 depicts the males that have these extreme shape values. The one on the right clearly has a centrally obese condition. The male on the left, though having a large abdominal area, has most of his excess weight centered at or below his hips and buttocks area (a frontal view would help the reader see this more clearly).

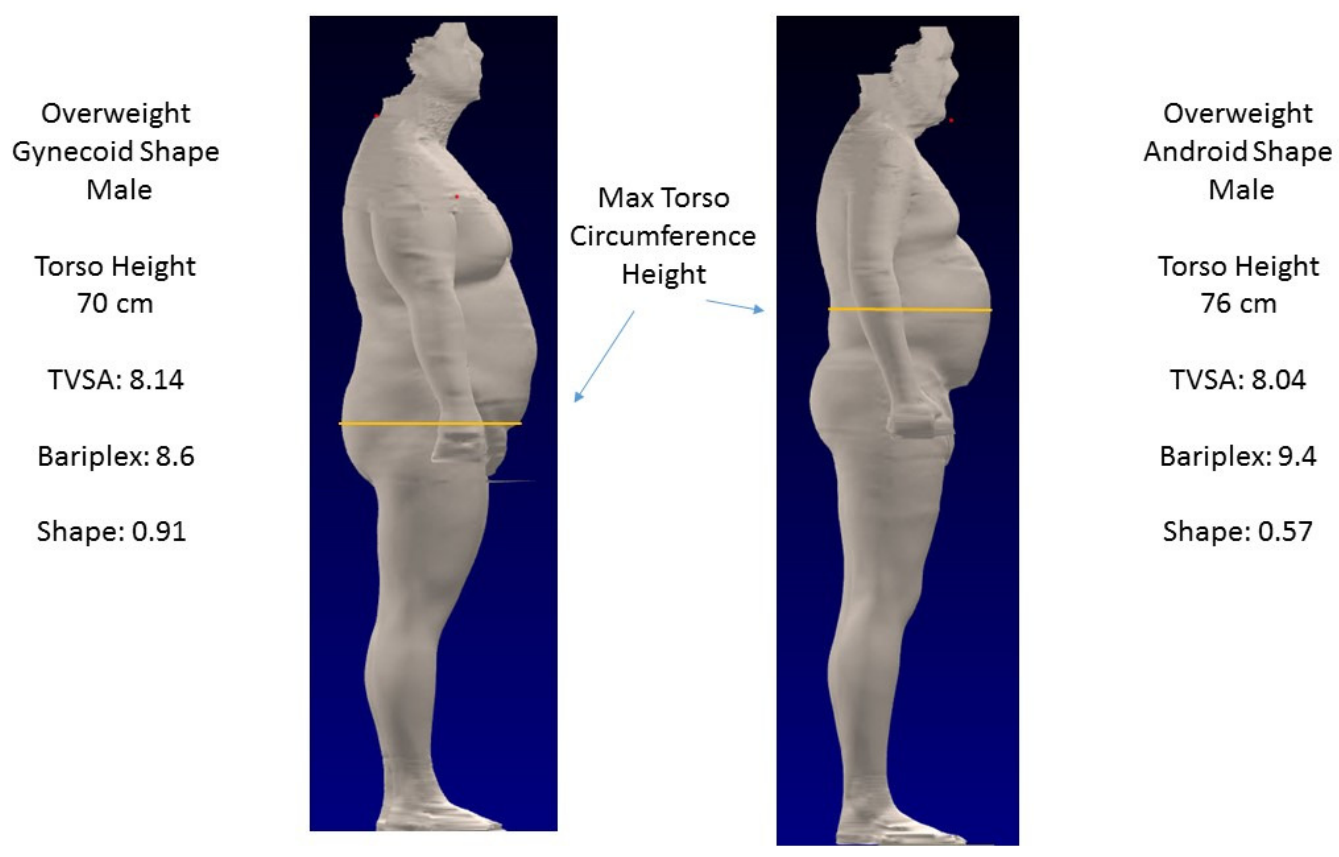

Figure 11. Example of Gynecoid and Android Obese Males Shapes

\section{Summary analysis for the male subgroups}

Table 6 compares the averages of the three groups of males analyzed: Thin, Borderline and Obese.

\begin{tabular}{|c|c|c|c|c|c|}
\hline Averages & TH & TVSA & Bplex & Shape & Mid-Max Dist. \\
\hline THIN & 75 & 6.89 & 10.88 & 0.92 & 27 \\
\hline Borderline & 74 & 7.55 & 9.8 & 0.9 & 26 \\
\hline Obese & 74 & 8.16 & 9.11 & 0.74 & 15 \\
\hline
\end{tabular}

Table 6. Summary of Group Averages for the Male Subpopulations 
The above table indicates that the average torso height of the males in the sample set was around 74 $\mathrm{cm}$. Torso height gives no indication as to amount of weight, torso volume or torso surface area an individual may possess. It is simply a linear measurement which spans from the "Back of the Neck" landmark to an individual's "Crotch Point," as measured by the scanner software. There is some effect however, on the "amount" of torso volume and torso surface area an individual may have in that the torso height is reflective of the height of the individual. The greater the torso height, in general, the taller the man. Dividing the TVSA into the Torso Height to produce the Bariplex is an attempt to normalize a "space indicator" over the range of torso height possibilities.

The TVSA and the Bariplex give no indication as to the distribution of weight in the subject's torso. That is found using the Shape Descriptor formula. Males who possess high Bariplex values are ordinarily thin and well proportioned. A shape value of .90 to 1.00 indicates that their maximum torso circumference height from the back of their neck is nearly the same or the same as their overall torso height and thus have no detectable excess weight distribution. Those who possess a high Bariplex and a shape value of .90 to 1.00 are definitely thin and well proportioned. An individual who possesses a high Bariplex value, and a shape descriptor value of less than 0.8 has some sort of exaggerated weight distribution.

Males that have a Bariplex value of between 9.5 and 10.0 have a tendency to be slightly overweight or heavier. The shape descriptor can be utilized to determine the individual's shape characteristic.

There is a certain point where an individual can be considered overweight or obese by various degrees. These individuals have a Bariplex of 9.5 or lower. Their body shape is differentiated from the thin and borderline in that there is a noticeable distribution of excess fat as indicated by the value of the primary shape descriptor. The lower the primary shape value, the more centrally obese the individual.

As a corollary, those that appear thin and shapely generally have a greater distance between their torso height mid-point and their maximum torso height circumference, given similar torso height. As individuals become centrally obese, this distance begins to decrease. In general, the smaller the distance between these two heights, the more centrally obese the individual.

\section{Analysis of Female Population}

The summary statistics for the female population are found Table 7.

\begin{tabular}{|c|c|c|c|c|c|}
\hline Summary & $\begin{array}{c}\text { Torso Height } \\
\mathrm{cm}\end{array}$ & TVSA & Bariplex & Shape & $\begin{array}{c}\text { Mid-Max Dist. } \\
\mathrm{cm}\end{array}$ \\
\hline Median & 65 & 6.41 & 9.53 & 0.87 & 25 \\
\hline Low & 57 & 5.5 & 6.51 & 0.77 & 16 \\
\hline High & 76 & 10.28 & 11.98 & 0.95 & 32 \\
\hline Average & 65 & 6.96 & 9.66 & 0.87 & 24 \\
\hline STD DEV & 5 & 1.36 & 1.53 & 0.06 & 5 \\
\hline
\end{tabular}

Table 7. Summary Statistics for Female Population

Inspection of the table above yields two immediate observations. One is that the population of women had an average torso height $6 \mathrm{~cm}$ less than their male counterparts. This is shown graphically in Figure 2. The other observation is that no women possessed a shape that was considered "android," or centrally obese. In general, if there was any excess weight on this set of females, it was distributed at or near the hips area as indicated by the Shape descriptor value.

Example ranges of female shape and size that were analyzed are arrayed in the Figure 12. For illustrative purposes they have been scaled to appear as if they were all the same height. In actuality, there are some variances in individual torso heights, as per the normal population. 


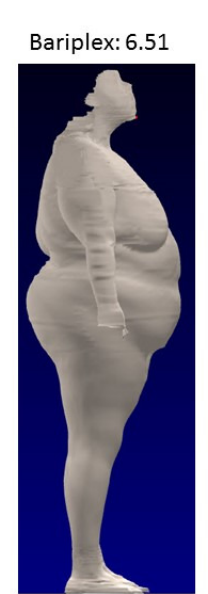

Morbidly Obese

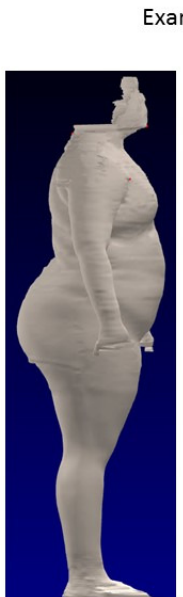

Example of the Range of Female Shapes and Sizes Analyzed

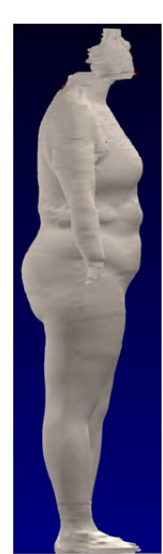

Obese

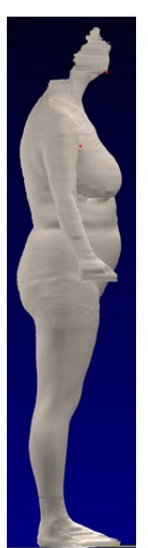

Overweight
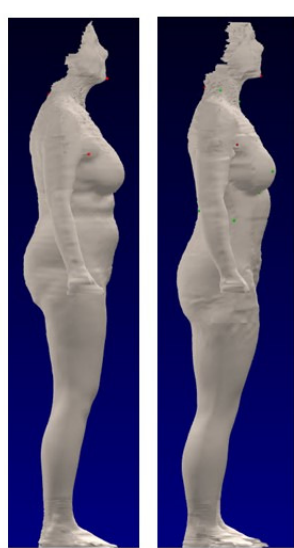

Proportional
Bariplex: 11.98

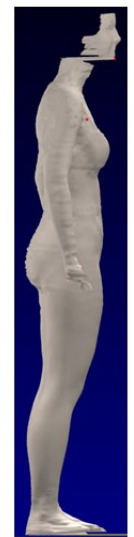

Thin

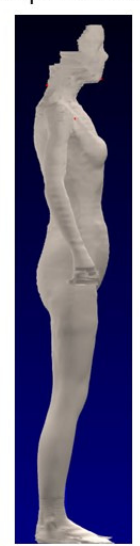

Very Thin

Figure 12. Representative Shapes for the Female Sample Population

\section{Analysis of the thin, or well-proportioned female sub-population}

For convenience of analysis, females that possessed a Bariplex value of 10.0 or higher were grouped into the "thin" category. Their statistics are summarized in Table 8.

\begin{tabular}{|c|c|c|c|c|c|}
\hline Thin & $\begin{array}{c}\text { Torso Height } \\
\mathrm{cm}\end{array}$ & TVSA & Bariplex & Shape & $\begin{array}{c}\text { Mid-Max Dist. } \\
\mathrm{cm}\end{array}$ \\
\hline Median & 65 & 6 & 11.32 & 0.93 & 28 \\
\hline Low & 60 & 5.5 & 10.11 & 0.81 & 18 \\
\hline High & 71 & 6.32 & 11.98 & 0.95 & 32 \\
\hline Average & 66 & 5.88 & 11.20 & 0.91 & 27 \\
\hline STD DEV & 3 & 0.30 & 0.62 & 0.05 & 4 \\
\hline
\end{tabular}

Table 8. Summary Statistics for Female Subpopulation having Bariplex Value of 10.0 or Greater

The average Bariplex value for the group of thin women was 11.20, with one example reaching 11.98 , a value usually associated with the very thin. This is the body model in the far right of in Figure 12.

All the women in this Bariplex category possessed an "hourglass" figure to a varying extent. Even the visually thinnest female possessed a waist-to-hips ratio of 0.74 as measured by the scanner software. In general, if a female possesses a Bariplex of 10 or greater and a Primary Shape value of .80 or greater she is visually thin or well-proportioned. There appear to be no exceptions. A Bariplex value of 11.5 or higher is a very thin female.

\section{Analysis of the "borderline trending overweight" group of women}

The "borderline trending overweight" group was selected out of convenience to have a Bariplex value of between 9.00 and 9.99. Here we begin to see the average shape of the group begin to change from .90 or higher associated with the group of thin women, to below 0.90 indicating that there is some excess fat distribution, centered particularly around the hips area. All of these women, when compared to their thin counterparts were either slightly or noticeably overweight. Their summary statistics are tabulated and presented in Table 9.

\begin{tabular}{|c|c|c|c|c|c|}
\hline Borderline & $\begin{array}{c}\text { Torso Height } \\
\text { cm }\end{array}$ & TVSA & Bariplex & Shape & $\begin{array}{c}\text { Mid-Max Dist. } \\
\text { cm }\end{array}$ \\
\hline Median & 62 & 6.67 & 9.32 & 0.87 & 24 \\
\hline Low & 57 & 5.93 & 9.05 & 0.77 & 16 \\
\hline High & 69 & 7.58 & 9.61 & 0.93 & 26 \\
\hline Average & 63 & 6.71 & 9.32 & 0.86 & 23 \\
\hline STD DEV & 5 & 0.61 & 0.25 & 0.06 & 4 \\
\hline
\end{tabular}

Table 9. Summary Statistics for Female Subpopulation having a Bariplex Value between 9.00 and 9.99 
Example of two females in the "borderline trending overweight" group can be found in the center right of Figure 12.

\section{Analysis of overweight female examples}

Lastly, those females who had a Bariplex value of under 9.0 were categorically obese. The lower the Bariplex value, the more obese the female. The summary statistics for this group of females can be found in Table 10.

\begin{tabular}{|c|c|c|c|c|c|}
\hline Obese & $\begin{array}{c}\text { Torso Height } \\
\mathrm{cm}\end{array}$ & TVSA & Bariplex & Shape & $\begin{array}{c}\text { Mid-Max Dist. } \\
\mathrm{cm}\end{array}$ \\
\hline Median & 66 & 8.17 & 8.36 & 0.805 & 19 \\
\hline Low & 62 & 6.9 & 6.51 & 0.78 & 19 \\
\hline High & 76 & 10.28 & 8.99 & 0.89 & 25 \\
\hline Average & 67 & 8.38 & 8.10 & 0.82 & 20 \\
\hline STD DEV & 5 & 1.22 & 0.92 & 0.04 & 2 \\
\hline
\end{tabular}

Table 10. Summary Statistics for Female Subpopulation having a Bariplex Value below 9.00

Examples of obese women can be found on the far left of the figures arrayed in Figure 12. The group of women classified as obese by their Bariplex value each had sizeable excess weight distributed, in these instances, predominantly around their hips and buttocks area. This is indicated by their Shape Descriptor value being at or near 0.80 . In practice, we have encountered morbidly obese female weight loss surgery candidates that possess mixed shapes, and even near-android shapes. Their shape would be reflected by their Shape Descriptor value being between 0.79 and 0.7 , with the lower Shape Descriptor value being associated with a higher degree of centralized obesity.

Figure 13 examines two females in the sample set that had the same torso height. The female on the left is considered to be overweight. The female on the right is considered to be thin. The overweight female has a higher TVSA, indicating that her torso volume is proportionally greater than her torso surface area. She takes up more "space." The female on the right has a surface area proportionally greater than her torso volume. As can be seen, she takes up less "space." When the Shape Descriptor formula is applied to the overweight female, it finds that her maximum torso height circumference is at or near her hips circumference height location. This indicates that the preponderance of excess weight is centered around her hips and buttocks area. When the same formula is applied to the thin female, it finds that her maximum torso height circumference is below her hips area, meaning, in essence, that it couldn't find any distortions in the shape along her torso contours that would indicate excess fat distribution.

Females with Same Torso Height but Different Size
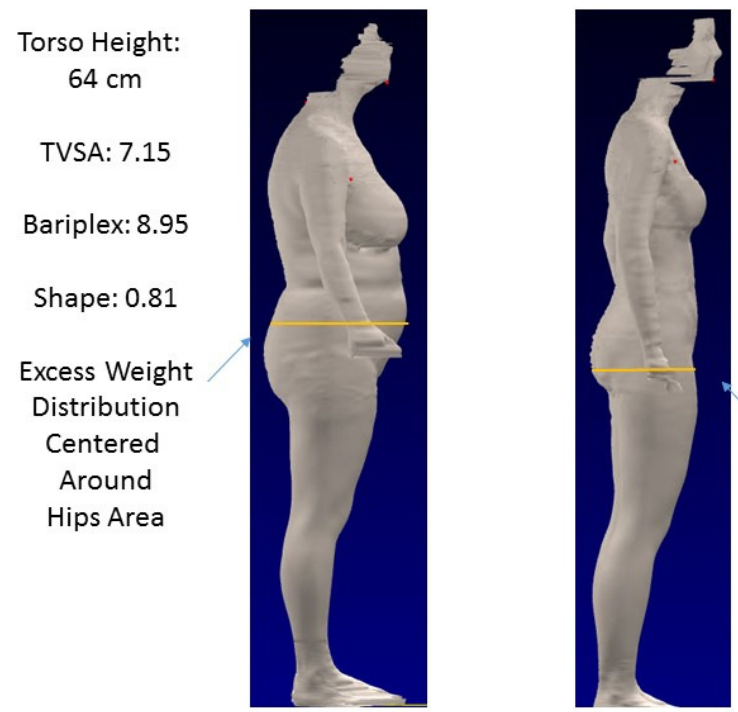

Torso Height: $64 \mathrm{~cm}$

TVSA: 5.57

Bariplex: 11.5

Shape: 0.93

No Excess Weight

Shape Indicator

Not Functioning

Figure 13. Two Females with Same Torso Heights and Different Body Shapes 


\section{Summary of Female Groups}

Table 11 contains the summary statistics for the female subpopulations.

\begin{tabular}{|c|c|c|c|c|c|}
\hline Averages & $\begin{array}{c}\text { Torso Height } \\
\mathrm{cm}\end{array}$ & TVSA & Bariplex & Shape & $\begin{array}{c}\text { Mid-Max Dist. } \\
\mathrm{cm}\end{array}$ \\
\hline Thin & 66 & 5.88 & 11.2 & 0.91 & 27 \\
\hline Borderline & 63 & 6.71 & 9.32 & 0.86 & 23 \\
\hline Obese & 67 & 8.38 & 8.1 & 0.82 & 20 \\
\hline
\end{tabular}

Table 11, Summary Statistics for the Female Subpopulations

Inspection of the summary of averages for the three groups of women generally follows the same pattern as the men, but the change in the shape descriptors as the females become obese is not as dramatic as the change of the shape descriptors for the overweight men.

Men and women that have a Bariplex value that places them in the thin category, and a shape descriptor value of 0.9 or greater are categorically thin and well-proportioned, or, if the Bariplex value is very high, even "dangerously" thin.

However, as men begin to gain weight there is a tendency to store the excess fat around the abdominal area. This tendency is reflected in the relatively rapid change in the shape indicator value as the difference between the overall torso height and the torso height from the back of the neck to the maximum torso height circumference begins to move upward. Women, on the other hand, tend to store their excess weight around their hips and buttocks area. The shape indicator appears to change for women at a slower rate due this phenomenon, but it is only because the contours of the female torso that generally become distorted due to excess weight are located around this area.

\section{Conclusion}

The torso height measurement is one thing humans all have in common. It can be considered to be fixed once adulthood is reached. The torso height is range bound in the adult population. For a fixed torso height, there is a relationship between its volume and surface area such that the greater the proportion of volume over surface area, the more "space" an individual occupies and hence the more obese the individual. Proportionally well-built and thin individuals have a low torso volume/torso surface area ratio (TVSA) regardless of gender. Visually obese individuals have a higher TVSA value.

Dividing the TVSA of an individual by their torso height yields a dimensionless value that is called the Bariplex. This attempts to normalize the TVSA so it can be compared across the range of torso heights. An interpretive scale was developed such that one's Bariplex falls into a descriptive category.

Both the TVSA and the Bariplex are indications of the amount of adiposity an individual possesses, but neither of them give any indication as to the distribution of the excess fat. This must be found by using the Shape Descriptor formula. The Shape Descriptor formula uses information in the torso slice file provided by the body scanner software to locate the height of the maximum torso circumference. This indicates where the largest concentration of excess fat along the torso is located. It also finds the median (midpoint) height of the torso. If the maximum torso slice height is closer to the midpoint, that individual has excess fat concentrated at or near the abdominal area. If the maximum torso slice height is at or near or the hips circumference height area, that individual has excess fat distributed predominantly around their hips and buttocks.

The shape descriptor formula was designed to mathematically differentiate the shape of obese to morbidly obese individuals. Thin males and females do not have any excess fat and are not obese. When the shape descriptor formula is applied to these body models, it returns a value outside of its normal range. This is valuable information as it indicates that at some point during weight gain, the Shape Descriptor formula begins to detect exaggerations in body shape, and indicates the location of the excess fat distribution.

The combination of the Bariplex, a measure of how much "space" one occupies, and the Shape Descriptor formula, combine in such a way as to provide early warning as to the onset of an overweight condition. This could be particularly important for men, as men tend to store excess fat around the abdominal region. The information required to calculate the Bariplex and the shape of the individual is provided by a 3D body scanner, and measurement software that is widely available across the industry. These adiposity and shape formulas use a 3D scanner to its full potential in that linear and circumferential measurements, along with the height of those measurements combine with 
surface area and volume information to give an indication of the level of obesity of an individual, and, if obese, where the concentration of excess weight is likely to be distributed.

Routine 3D body scanning can establish a trend that can be monitored on an on-going basis. These formulas can be part of a measurement profile applied to the scanner body model and appear on a measurement printout alongside common circumferential measurements. Moreover, since both the size of the individual and the shape of the individual are in the form of numbers, they can be evaluated remotely without the need for visual confirmation of observing either the individuals themselves or their body models.

\section{References}

1) Complete Spacial Evaluation of the Preoperative Bariatric Patient: New Insights into Body Composition. Stephen D. Wohlgemuth, M.D., David Stefan, M.S.E.E. Paper ID 532013 3D Body Scanning Conference. Long Beach, CA. USA. October 2013. doi: http://dx.doi.org/10.15221/13.340

2) Theory and Practical Steps to Introducing a new 3D Public Health Indicator to replace BMI Using Existing Population-based Multidimensional Reference Measurement Sets. David B. Stefan, M.S.E.E, Stephen D. Wohlgemuth, M.D., David A. Gilbert, M.D. Paper ID 552014 3D Body Scanning Conference. Lugano, CH. October 2014. doi:10.15221/14.299.

3) Integrating a 3D Body Scanner into an Active Bariatric Surgery Clinic: Practical Experiences, History, Tips and Pitfalls. David B. Stefan, M.S.E.E., Stephen D. Wohlgemuth, M.D., David A. Gilbert, M.D. Paper ID 562014 3D Body Scanning Conference. Lugano, CH. October 2014. doi:10.15221/14.124.

4) Breast Augmentation Virtual Surgery Using 3D Body Scanning: Bridging the Gap between Patient Expectations and Surgical Practicalities. David A. Gilbert, M.D., David B. Stefan, M.S.E.E. Paper ID 512013 3D Body Scanning Conference. Long Beach, CA. USA. 2013. doi: http://dx.doi.org/10.15221/13.285

5) Categorizing the Morbidly Obese Body Shape and Estimating Body Appearance Outcome before Weight Loss Surgery using 3D Anthropometric Data. David B. Stefan, M.S.E.E; David A. Gilbert, M.D. Paper ID 102015 3D Body Scanning Conference. Lugano, CH. October 2015. doi:10.15221/15.038. 